\title{
Matrix Representation of Soft Topology
}

\author{
${ }^{1}$ P.Rohini devi and ${ }^{2}$ Dr.R.Padmapriya \\ P.G. and Research Department of Mathematics,
}

Erode Arts and Science College (Autonomous), Erode-09, Tamilnadu, India.

${ }^{1}$ Email: rohinidevi83@gmail.com

${ }^{2}$ Email: priyabharathi28@gmail.com

\begin{abstract}
The purpose of this paper is to represent a soft topology by matrices over $\{0,1\}$ and to characterize soft topology by using matrices. Further a data set consisting of binary matrices for diabetic patients will be created for a particular soft topology on the collection of diabetic patients and a method for the recommendations of a doctor to diabetic patients is given using the above data set. More over an algorithm has been created to choose the right recommendation for a patient.
\end{abstract}

Kew words: soft set, soft topology, soft matrix, soft addition, soft multiplication.

\section{Introduction}

Molodtsov [6] is a pioneer in studying soft sets to deal with the vague concepts associated with real life problems. Following this, several mathematicians developed soft set theory in various directions [ 7,14]. Maji and Roy[5] used tabular representation of soft sets along with rough sets [10] in a decision making problem. Topologists $[1-4,8,9,11,12,13,15]$ extended the notion of topology to soft sets and studied the basic properties of soft topology. In this paper, soft sets and soft topology are characterized using matrices over $\{0,1\}$. Moreover a data set consisting of binary matrices for diabetic patients will be created for a particular soft topology on the collection of diabetic patients and a method for the recommendations of a doctor to diabetic patients is given using the above data set. In this regard, an algorithm is given to implement this discussion.

\section{Preliminaries}

Let $\mathrm{X}$ be a non empty set and $\mathrm{E}$ be a parameter space. If $\mathrm{A} \subseteq \mathrm{E}$ and $\mathrm{F}: \mathrm{A} \rightarrow 2^{\mathrm{X}}$ then the pair $(\mathrm{F}, \mathrm{A})$ is called a soft subset of $X$ over the parameter space. Let $S(X, E)$ be the collection of all soft subsets of $X$ with parameter space $E$. The pair $(\mathrm{F}, \mathrm{A})$ can be identified with $(\mathrm{F}, \mathrm{E})=\tilde{F}$ by defining $\mathrm{F}(\mathrm{e})=\varnothing$ for all $\mathrm{e} \in \mathrm{E} \backslash \mathrm{A}$. Let $\widetilde{\varnothing}$ and $\tilde{X}$ denote the null soft subset and absolute soft subset of $X$ defined by $\widetilde{\varnothing}(e)=\varnothing$ and $\tilde{X}(e)=\mathrm{X}$ for all $\mathrm{e} \in \mathrm{E}$. Let $\tilde{F}$ and $\tilde{G}$ be any two soft sets in $\mathrm{S}(\mathrm{X}, \mathrm{E})$. We say that $\tilde{F} \subseteq \tilde{G}$ if $\tilde{F}(\mathrm{e})$ $\subseteq \tilde{G}(\mathrm{e})$ for all $\mathrm{e} \in \mathrm{E}$ and $\tilde{F} \sqsupseteq \tilde{G}$ if $\tilde{G} \subseteq \tilde{F}$. The soft sets $\tilde{F} \sqcap \tilde{G}$ and $\tilde{F} \sqcup \tilde{G}$ respectively denote the soft intersection and soft union of $\tilde{F}$ and $\tilde{G}$. The soft set $\tilde{F} \boxminus \tilde{G}$ is the soft difference of $\tilde{G}$ from $\tilde{F}$. In particular $\tilde{X} \boxminus \tilde{G}$ is the soft complement of $\tilde{G}$. A sub collection $\tilde{\tau}$ of $\mathrm{S}(\mathrm{X}, \mathrm{E})$ is a soft topology on $\mathrm{X}$ if (i) $\widetilde{\emptyset} \in \tilde{\tau}$,(ii) $\tilde{X} \in \tilde{\tau}$, (iii) $\tilde{\tau}$ is closed under finite soft intersection and (iv) $\tilde{\tau}$ is closed under arbitrary soft union.

If $\tilde{\tau}$ is a soft topology on $X$ then the triplet $(X, \tilde{\tau}, E)$ is called a soft topological space and $\tilde{\tau}$ is a soft topology over $(\mathrm{X}, \mathrm{E})$. The members of $\tilde{\tau}$ are called soft open in $(\mathrm{X}, \tilde{\tau}$, E). The soft complement of a soft open set is soft closed.

\section{Matrix Representation}

Most of the sets and associated space of parameter elements in real life applications are finite sets. Therefore we assume that $\mathrm{X}$ is a finite set of discourse with finite parameter space $\mathrm{E}$. Let $\mathrm{X}=\left\{\mathrm{x}_{1}, \mathrm{x}_{2}, \mathrm{x}_{3}, \ldots, \mathrm{x}_{\mathrm{m}}\right\}$ and $\mathrm{E}=\left\{\mathrm{e}_{1}, \mathrm{e}_{2}, \mathrm{e}_{3}, \ldots, \mathrm{e}_{\mathrm{n}}\right\}$. If $\tilde{F}$ is a soft subset in $\mathrm{S}(\mathrm{X}, \mathrm{E})$ then it can be identified by a matrix $\operatorname{Mat}(\tilde{F})=\left[\mathrm{f}_{\mathrm{ij}}\right]$ with $\mathrm{m}$ rows and $\mathrm{n}$ columns where $\mathrm{f}_{\mathrm{ij}}=1$ if $\mathrm{x}_{\mathrm{i}} \in \tilde{F}\left(\mathrm{e}_{\mathrm{j}}\right)$ and $=0$ if $\mathrm{x}_{\mathrm{i}} \notin \tilde{F}\left(\mathrm{e}_{\mathrm{j}}\right)$. To characterize the properties of soft sets, the following operations on $\{0,1\}$ are used. If $x, y \in\{0,1\}$ then $x \oplus y=\left\{\begin{array}{c}0 \text { if } x=y=0 \\ 1 \text { otherwise }\end{array}\right.$ and $x \odot y=\left\{\begin{array}{c}1 \text { if } x=y=1 \\ 0 \text { otherwise }\end{array}\right.$. If $\left[\mathrm{f}_{\mathrm{ij}}\right]$ and $\left[\mathrm{g}_{\mathrm{ij}}\right]$ are two matrices over $\{0,1\}$ then their addition and multiplication are defined component wise that is $\left[\mathrm{f}_{\mathrm{ij}}\right] \oplus\left[\mathrm{g}_{\mathrm{ij}}\right]=\left[\mathrm{f}_{\mathrm{ij}} \oplus \mathrm{g}_{\mathrm{ij}}\right]$ and $\left[\mathrm{f}_{\mathrm{ij}}\right] \odot\left[\mathrm{g}_{\mathrm{ij}}\right]=\left[\mathrm{f}_{\mathrm{ij}} \odot \mathrm{g}_{\mathrm{ij}}\right]$. The following lemmas will be useful in sequel. Throughout this paper $\tilde{F}$ and $\tilde{G}$ are soft sets in $\mathrm{S}(\mathrm{X}, \mathrm{E}), \operatorname{Mat}(\tilde{F})=\left[\mathrm{f}_{\mathrm{ij}}\right]$ and $\operatorname{Mat}(\tilde{G})=\left[\mathrm{g}_{\mathrm{ij}}\right]$. Let $\operatorname{Mat}(\{0,1\})$ be the collection of matrices over $\{0,1\}$ The next two lemmas show that the operations $\oplus$ and $\odot$ are well defined.

Lemma 3.1: $\quad \operatorname{Mat}(\tilde{F}) \oplus \operatorname{Mat}(\tilde{G})=\left[\mathrm{f}_{\mathrm{ij}} \oplus \mathrm{g}_{\mathrm{ij}}\right] \quad$ and $\operatorname{Mat}(\tilde{F}) \odot \operatorname{Mat}(\tilde{G})=\left[\mathrm{f}_{\mathrm{ij}} \odot \mathrm{g}_{\mathrm{ij}}\right]$.

Lemma 3.2: If $\left[h_{i j}\right] \in \operatorname{Mat}(\{0,1\})$ then there is a unique soft set $\widetilde{H}$ such that $\operatorname{Mat}(\widetilde{H})=\left[\mathrm{h}_{\mathrm{ij}}\right]$.

The above lemma motivates to extend operations $\oplus$ and $\odot$ on soft sets. For any two soft sets $\tilde{F}$ and $\tilde{G}$, $\operatorname{Mat}(\tilde{F}) \oplus \operatorname{Mat}(\tilde{G})$ and $\operatorname{Mat}(\tilde{F}) \odot \operatorname{Mat}(\tilde{G})$ are matrices over $\{0,1\}$. Then by using Lemma 3.2 , there are softs $\widetilde{H}$ and $\widetilde{K}$ such that $\operatorname{Mat}(\widetilde{F}) \oplus \operatorname{Mat}(\tilde{G})=\operatorname{Mat}(\widetilde{H})$ and $\operatorname{Mat}(\tilde{F}) \odot \operatorname{Mat}(\tilde{G})=\operatorname{Mat}(\widetilde{K})$ where $\widetilde{H}$ is uniquely determined by $\tilde{F}, \tilde{G} \& \operatorname{Mat}(\tilde{F}) \oplus \operatorname{Mat}(\tilde{G})$ and $\widetilde{K}$ is uniquely determined by $\tilde{F}, \tilde{G} \& \operatorname{Mat}(\tilde{F}) \odot \operatorname{Mat}(\tilde{G})$. Such soft sets $\widetilde{H}$ and $\widetilde{K}$ will be denoted by $\widetilde{F} \oplus \widetilde{G}=\widetilde{H}$ and $\tilde{F} \odot \tilde{G}=\widetilde{K}$. The soft sets $\tilde{F} \oplus \tilde{G}$ and $\tilde{F} \odot \tilde{G}$ are respectively known as soft addition and soft multiplication of soft sets $\tilde{F}$ and $\tilde{G}$.

The above discussion leads to the following lemma.

Lemma 3.3: $\operatorname{Mat}(\tilde{F} \oplus \tilde{G})=\operatorname{Mat}(\tilde{F}) \oplus \operatorname{Mat}(\tilde{G}) \quad$ and $\operatorname{Mat}(\tilde{F} \odot \tilde{G})=\operatorname{Mat}(\tilde{F}) \odot \operatorname{Mat}(\tilde{G})$. 
The above three lemmas have been illustrated in the following example.

Example 3.4: Let $U=\{$ Anitha, Murugesan, Sheeba, Thamizharasi, Selvi, Bageerathi, Manoharan, Usha, Sutha, Xavier \} be the list of students in a class whose academic advisor is Mr.X. The advisor used the parameters $\mathrm{e}_{1}=$ 'Bright', $\mathrm{e}_{2}=$ 'Intelligent', $\mathrm{e}_{3}=$ 'Weak', $\mathrm{e}_{4}=$ 'Dull', $\mathrm{e}_{5}=$ 'Average' to form the groups for his academic coaching. We use the notations $\mathrm{x}_{1}=$ 'Anitha', $\mathrm{x}_{2}=$ 'Murugesan', $\quad \mathrm{x}_{3}=$ 'Sheeba', $\quad \mathrm{x}_{4}=$ 'Thamizharasi', $\mathrm{x}_{5}={ }^{\prime}$ 'Selvi', $\mathrm{x}_{6}={ }^{\prime}$ Bageerathi', $\mathrm{x}_{7}={ }^{\prime}$ Manoharan', $\mathrm{x}_{8}={ }^{\prime}$ 'Usha', $\mathrm{x}_{9}=$ 'Sutha' and $\mathrm{x}_{10}=$ 'Xavier'. Therefore $\mathrm{E}=\left\{\mathrm{e}_{1}, \mathrm{e}_{2}, \mathrm{e}_{3}, \mathrm{e}_{4}\right.$, $\left.\mathrm{e}_{5}\right\}$ and $\mathrm{U}=\left\{\mathrm{x}_{1}, \mathrm{x}_{2}, \mathrm{x}_{3}, \mathrm{x}_{4}, \mathrm{x}_{5}, \mathrm{x}_{6}, \mathrm{x}_{7}, \mathrm{x}_{8}, \mathrm{x}_{9}, \mathrm{x}_{10}\right\}$. If $\tilde{F}=\left\{\left(\mathrm{e}_{1},\left\{\mathrm{x}_{7}, \mathrm{x}_{10}\right\}\right),\left(\mathrm{e}_{2},\left\{\mathrm{x}_{2}, \mathrm{x}_{6}, \mathrm{x}_{7}, \mathrm{x}_{10}, \mathrm{x}_{5}\right\}\right),\left(\mathrm{e}_{3},\left\{\mathrm{x}_{1}\right.\right.\right.$, $\left.\left.\left.\mathrm{x}_{4}\right\}\right),\left(\mathrm{e}_{4}, \varnothing\right),\left(\mathrm{e}_{5},\left\{\mathrm{x}_{3}, \mathrm{x}_{8}\right\}\right)\right\}$ and

$\tilde{G}=\left\{\left(\mathrm{e}_{1},\left\{\mathrm{x}_{2}, \mathrm{x}_{10}\right\}\right),\left(\mathrm{e}_{2},\left\{\mathrm{x}_{9}, \mathrm{x}_{7}, \mathrm{x}_{10}\right\}\right),\left(\mathrm{e}_{3},\left\{\mathrm{x}_{8}, \mathrm{x}_{6}\right\}\right),\left(\mathrm{e}_{4}\right.\right.$, $\left.\left.\left\{\mathrm{x}_{4}\right\}\right),\left(\mathrm{e}_{5},\left\{\mathrm{x}_{5}, \mathrm{x}_{1}\right\}\right)\right\}$ then

It is easy to verify the following identities.

$$
\begin{aligned}
& \operatorname{Mat}(\tilde{F} \oplus \tilde{G})=\operatorname{Mat}(\tilde{F}) \oplus \operatorname{Mat}(\tilde{G})= \\
& {\left[\begin{array}{lllll}
0 & 0 & 1 & 0 & 1 \\
1 & 1 & 0 & 0 & 0 \\
0 & 0 & 0 & 0 & 1 \\
0 & 0 & 1 & 1 & 0 \\
0 & 1 & 0 & 0 & 1 \\
0 & 1 & 1 & 0 & 0 \\
1 & 1 & 0 & 0 & 0 \\
0 & 0 & 1 & 0 & 1 \\
0 & 1 & 0 & 0 & 0 \\
1 & 1 & 0 & 0 & 0
\end{array}\right] \quad \text { and }}
\end{aligned}
$$

$\operatorname{Mat}(\tilde{F} \odot \tilde{G})=\operatorname{Mat}(\tilde{F}) \odot \operatorname{Mat}(\tilde{G})=\left[\begin{array}{ccccc}0 & 0 & 0 & 0 & 0 \\ 0 & 0 & 0 & 0 & 0 \\ 0 & 0 & 0 & 0 & 0 \\ 0 & 0 & 0 & 0 & 0 \\ 0 & 0 & 0 & 0 & 1 \\ 0 & 0 & 0 & 0 & 0 \\ 0 & 1 & 0 & 0 & 0 \\ 0 & 0 & 0 & 0 & 0 \\ 0 & 0 & 0 & 0 & 0 \\ 1 & 1 & 0 & 0 & 0\end{array}\right]$

The soft topologies are characterized by the operations $\oplus$ and $\odot$ as shown in the next theorem.

\section{Theorem 3.5:}

Let $\tilde{\tau}$ be a collection of soft sets in S(X,E) and $\mathrm{M}(\tilde{\tau})=\{\operatorname{Mat}(\tilde{F}) \in \operatorname{Mat}(\{0,1\}): \tilde{F} \in \tilde{\tau}\}=$ the collection of matrices of soft sets in $\tilde{\tau}$. Then $\tilde{\tau}$ is a soft topology if and only if the mathematical structures $(\mathrm{M}(\tilde{\tau}), \oplus)$ and $(\mathrm{M}(\tilde{\tau}), \odot)$ are monoids.

Proof: Suppose $\tilde{\tau}$ is a soft topology on $\mathrm{X}$.

$\widetilde{\emptyset} \in \tilde{\tau} \Rightarrow \operatorname{Mat}(\widetilde{\varnothing})=\left[\varphi_{\mathrm{ij}}\right] \in \mathrm{M}(\tilde{\tau})$ where $\varphi_{\mathrm{ij}}=0$ for $\mathrm{i}=1,2, \ldots, \mathrm{m}$ and $\mathrm{j}=1,2, \ldots, \mathrm{n}$.
$\operatorname{Mat}(\tilde{F})=\left[\begin{array}{ccccc}0 & 0 & 1 & 0 & 0 \\ 0 & 1 & 0 & 0 & 0 \\ 0 & 0 & 0 & 0 & 1 \\ 0 & 0 & 1 & 0 & 0 \\ 0 & 1 & 0 & 0 & 0 \\ 0 & 1 & 0 & 0 & 0 \\ 1 & 1 & 0 & 0 & 0 \\ 0 & 0 & 0 & 0 & 1 \\ 0 & 0 & 0 & 0 & 0 \\ 1 & 1 & 0 & 0 & 0\end{array}\right]$ and $\operatorname{Mat}(\tilde{G})=$
$\left[\begin{array}{lllll}0 & 0 & 0 & 0 & 1 \\ 1 & 0 & 0 & 0 & 0 \\ 0 & 0 & 0 & 0 & 0 \\ 0 & 0 & 0 & 1 & 0 \\ 0 & 0 & 0 & 0 & 1 \\ 0 & 0 & 1 & 0 & 0 \\ 0 & 1 & 0 & 0 & 0 \\ 0 & 0 & 1 & 0 & 0 \\ 0 & 1 & 0 & 0 & 0 \\ 1 & 1 & 0 & 0 & 0\end{array}\right]$.

$\tilde{X} \in \tilde{\tau} \Rightarrow \operatorname{Mat}(\tilde{X})=\left[\pi_{\mathrm{ij}}\right] \in \mathrm{M}(\tau)$ where $\pi_{\mathrm{ij}}=1$ for $\mathrm{i}=1,2, \ldots, \mathrm{m}$ and $\mathrm{j}=1,2, \ldots, \mathrm{n}$.

Let $\operatorname{Mat}(\tilde{F}), \operatorname{Mat}(\tilde{G}), \operatorname{Mat}(\widetilde{H}) \in \mathrm{M}(\tilde{\tau})$ where $\tilde{F}, \tilde{G}, \widetilde{H} \in \tilde{\tau}$. Since $\tilde{\tau}$ is a soft topology, it is clear that $\tilde{F} \sqcap \tilde{G}, \tilde{F} \sqcup \tilde{G}$ $\in \tilde{\tau} \quad$ so that $\operatorname{Mat}(\tilde{F} \sqcap \tilde{G}), \operatorname{Mat}(\tilde{F} \sqcup \tilde{G}) \in \operatorname{M}(\tilde{\tau})$. Let $\operatorname{Mat}(\tilde{F} \sqcap \tilde{G})=\left[\mathrm{h}_{\mathrm{ij}}\right]$.

Now $\mathrm{h}_{\mathrm{ij}}=1 \Leftrightarrow \mathrm{x}_{\mathrm{i}} \in(\tilde{F} \sqcap \tilde{G})\left(\mathrm{e}_{\mathrm{j}}\right) \Leftrightarrow \mathrm{x}_{\mathrm{i}} \in \tilde{F}\left(\mathrm{e}_{\mathrm{j}}\right)$ and $\mathrm{x}_{\mathrm{i}} \in \tilde{G}\left(\mathrm{e}_{\mathrm{j}}\right)$

$$
\Leftrightarrow \mathrm{f}_{\mathrm{ij}}=1 \text { and } \mathrm{g}_{\mathrm{ij}}=1 \Leftrightarrow \mathrm{f}_{\mathrm{ij}} \odot \mathrm{g}_{\mathrm{ij}}=1
$$

Also $\mathrm{h}_{\mathrm{ij}}=0 \Leftrightarrow \mathrm{xi} \notin(\tilde{F} \sqcap \tilde{G})\left(\mathrm{e}_{\mathrm{j}}\right)$

$\Leftrightarrow \quad\left[\mathrm{x}_{\mathrm{i}} \in \tilde{F}\left(\mathrm{e}_{\mathrm{j}}\right)\right.$ and $\left.\mathrm{x}_{\mathrm{i}} \notin \tilde{G}\left(\mathrm{e}_{\mathrm{j}}\right)\right]$ or $\left[\mathrm{x}_{\mathrm{i}} \notin \tilde{F}\left(\mathrm{e}_{\mathrm{j}}\right)\right.$ and $\left.\mathrm{x}_{\mathrm{i}} \in \tilde{G}\left(\mathrm{e}_{\mathrm{j}}\right)\right]$ or $\left[\mathrm{x}_{\mathrm{i}} \notin \tilde{F}\left(\mathrm{e}_{\mathrm{j}}\right)\right.$ and $\left.\mathrm{x}_{\mathrm{i}} \notin \tilde{G}\left(\mathrm{e}_{\mathrm{j}}\right)\right]$

$\Leftrightarrow\left[f_{i j}=1\right.$ and $\left.g_{i j}=0\right]$ or $\left[f_{i j}=0\right.$ and $\left.g_{i j}=1\right]$

or $\left[f_{i j}=0\right.$ and $\left.g_{i j}=0\right]$

$$
\Leftrightarrow \quad \mathrm{f}_{\mathrm{ij}} \odot \mathrm{g}_{\mathrm{ij}}=0 \text {. }
$$

Therefore $\quad \operatorname{Mat}(\tilde{F}) \odot \operatorname{Mat}(\tilde{G})=\left[\mathrm{f}_{\mathrm{ij}} \quad\right] \odot\left[\mathrm{g}_{\mathrm{ij}}\right]=\left[\mathrm{f}_{\mathrm{ij}}\right.$ ]$\odot\left[\mathrm{g}_{\mathrm{ij}}\right]=\left[\mathrm{f}_{\mathrm{ij}} \odot \mathrm{g}_{\mathrm{ij}}\right]=\left[\mathrm{h}_{\mathrm{ij}}\right]=\operatorname{Mat}(\tilde{F} \sqcap \tilde{G}) \in \mathrm{M}(\tilde{\tau})$. Also it is easy to prove that $(\operatorname{Mat}(\tilde{F}) \odot \operatorname{Mat}(\tilde{G})) \odot \operatorname{Mat}(\widetilde{H})=$ $\operatorname{Mat}(\widetilde{F}) \odot(\operatorname{Mat}(\tilde{G}) \odot \operatorname{Mat}(\widetilde{H}))$ and

$\operatorname{Mat}(\tilde{F}) \odot \operatorname{Mat}(\tilde{X})=\operatorname{Mat}(\tilde{X}) \odot \operatorname{Mat}(\tilde{F})=\operatorname{Mat}(\tilde{F})$. This proves that $(\mathrm{M}(\tilde{\tau}), \odot)$ is a monoid.

Lett $\operatorname{Mat}(\tilde{F} \sqcup \tilde{G})=\left[\mathrm{h}_{\mathrm{ij}}\right]$. Now

$$
\begin{aligned}
\mathrm{h}_{\mathrm{ij}}=0 \Leftrightarrow \mathrm{x}_{\mathrm{i}} \notin(\tilde{F} \sqcup \tilde{G})\left(\mathrm{e}_{\mathrm{j}}\right) & \Leftrightarrow \mathrm{x}_{\mathrm{i}} \notin \tilde{F}\left(\mathrm{e}_{\mathrm{j}}\right) \text { and } \mathrm{x}_{\mathrm{i}} \notin \tilde{G}\left(\mathrm{e}_{\mathrm{j}}\right) \\
& \Leftrightarrow \mathrm{f}_{\mathrm{ij}}=0 \text { and } \mathrm{g}_{\mathrm{ij}}=0 \Leftrightarrow
\end{aligned}
$$

$\mathrm{f}_{\mathrm{ij}} \oplus \mathrm{g}_{\mathrm{ij}}=0$.

Also $\mathrm{h}_{\mathrm{ij}}=1 \Leftrightarrow \mathrm{xi} \in(\tilde{F} \sqcup \tilde{G})\left(\mathrm{e}_{\mathrm{j}}\right)$

$$
\Leftrightarrow\left[\mathrm{x}_{\mathrm{i}} \in \tilde{F}\left(\mathrm{e}_{\mathrm{j}}\right) \text { and } \mathrm{x}_{\mathrm{i}} \notin \tilde{G}\left(\mathrm{e}_{\mathrm{j}}\right)\right] \text { or }\left[\mathrm{x}_{\mathrm{i}} \notin \tilde{F}\left(\mathrm{e}_{\mathrm{j}}\right)\right. \text { and }
$$
$\left.\mathrm{x}_{\mathrm{i}} \in \tilde{G}\left(\mathrm{e}_{\mathrm{j}}\right)\right]$ or $\left[\mathrm{x}_{\mathrm{i}} \in \tilde{F}\left(\mathrm{e}_{\mathrm{j}}\right)\right.$ and $\left.\mathrm{x}_{\mathrm{i}} \in \tilde{G}\left(\mathrm{e}_{\mathrm{j}}\right)\right]$

$$
\Leftrightarrow\left[f_{i j}=1 \text { and } g_{i j}=0\right] \text { or }\left[f_{i j}=0 \text { and } g_{i j}=\right.
$$
$1]$ or $\left[f_{i j}=1\right.$ and $\left.g_{i j}=1\right]$ 


$$
\Leftrightarrow \mathrm{f}_{\mathrm{ij}} \oplus \mathrm{g}_{\mathrm{ij}}=1
$$

Therefore $\quad \operatorname{Mat}(\tilde{F}) \oplus \operatorname{Mat}(\tilde{G})=\left[\mathrm{f}_{\mathrm{ij}} \quad\right] \oplus\left[\mathrm{g}_{\mathrm{ij}}\right]=\left[\mathrm{f}_{\mathrm{ij}}\right.$ ]$\oplus\left[\mathrm{g}_{\mathrm{ij}}\right]=\left[\mathrm{f}_{\mathrm{ij}} \oplus \mathrm{g}_{\mathrm{ij}}\right]=\left[\mathrm{h}_{\mathrm{ij}}\right]=\operatorname{Mat}(\tilde{F} \cup \tilde{G}) \in \mathrm{M}(\tilde{\tau})$.

Also it is easy to prove that $(\operatorname{Mat}(\tilde{F}) \oplus \operatorname{Mat}(\tilde{G})) \oplus \operatorname{Mat}(\widetilde{H})=$ $\operatorname{Mat}(\tilde{F}) \oplus(\operatorname{Mat}(\tilde{G}) \oplus \operatorname{Mat}(\widetilde{H})) \quad$ and $\quad \operatorname{Mat}(\tilde{F}) \oplus \operatorname{Mat}(\widetilde{\varnothing}$ )$=\operatorname{Mat}(\widetilde{\varnothing}) \oplus \operatorname{Mat}(\tilde{F})=\operatorname{Mat}(\tilde{F})$. This proves that $(\mathrm{M}(\tilde{\tau}), \oplus)$ is a Monoid.

Definition 3.6: If $\left[\mathrm{f}_{\mathrm{ij}}\right]$ is a matrix over $\{0,1\}$ then its complement is defined as

$\left[f_{i j}\right]^{c}=\left[g_{i j}\right]$ where $g_{i j}=0$ if $f_{i j}=1$ and $g_{i j}=1$ if $f_{i j}=0$.

The next lemma can be easily established.

Lemma 3.7: $\operatorname{Mat}(\tilde{X} \boxminus \tilde{F})=(\operatorname{Mat}(\tilde{F}))^{c}=\left[\mathrm{f}_{\mathrm{ij}}\right]^{\mathrm{c}}$.

Theorem 3.8: $\quad \tilde{F}$ is soft closed if and only if $(\operatorname{Mat}(\tilde{F}))^{\mathrm{c}} \in \mathrm{M}(\tilde{\tau})$.

Proof: $\quad \tilde{F}$ is soft closed $\Leftrightarrow \tilde{X} \boxminus \tilde{F} \in \tilde{\tau} \Leftrightarrow \operatorname{Mat}(\tilde{X} \boxminus$ $\tilde{F}) \in \mathrm{M}(\tilde{\tau})$

)$)^{\mathrm{c}} \in \mathrm{M}(\tilde{\tau})$

$$
\Leftrightarrow(\operatorname{Mat}(\tilde{F}
$$

\section{Application to Diabetic Patients}

Soft topological concepts can be used to analyse the diabetic patients for controlling their sugar level. Diabetic patients used some of the parameters namely $\mathrm{e}_{1}=$ 'regular walking', $\mathrm{e}_{2}=$ 'regular swimming', $\mathrm{e}_{3}=$ 'regular cycling', $\mathrm{e}_{4}=$ 'regular Yoga', $\mathrm{e}_{5}=$ 'diet control' and $\mathrm{e}_{6}=$ 'taking medicine' to maintain the sugar level at the desired range. Let $\mathrm{U}=\left\{\mathrm{P}_{1}, \mathrm{P}_{2}, \mathrm{P}_{3}, \mathrm{P}_{4}, \mathrm{P}_{5}, \mathrm{P}_{6}, \mathrm{P}_{7}, \mathrm{P}_{8}, \mathrm{P}_{9}, \mathrm{P}_{10}\right\}$ be the set of diabetic patients in a reputed hospital. The problem of a doctor is to suggest appropriate parameter for a patient to control the sugar level.

$\tilde{F}=\left\{\left(\mathrm{e}_{1},\left\{\mathrm{P}_{1}, \mathrm{P}_{5}, \mathrm{P}_{8}, \mathrm{P}_{10}\right\}\right),\left(\mathrm{e}_{2},\left\{\mathrm{P}_{2}, \mathrm{P}_{4}, \mathrm{P}_{9}\right\}\right),\left(\mathrm{e}_{3},\left\{\mathrm{P}_{3}\right.\right.\right.$, $\left.\left.\mathrm{P}_{6}, \mathrm{P}_{7},\right\}\right),\left(\mathrm{e}_{4},\left\{\mathrm{P}_{8}, \mathrm{P}_{9}, \mathrm{P}_{10}\right\}\right)$,

\})\}.

$$
\left(\mathrm{e}_{5},\left\{\mathrm{P}_{4}, \mathrm{P}_{5}, \mathrm{P}_{6}, \mathrm{P}_{7}\right\}\right),\left(\mathrm{e}_{6},\left\{\mathrm{P}_{1}, \mathrm{P}_{3}, \mathrm{P}_{5}, \mathrm{P}_{7}, \mathrm{P}_{9}\right.\right.
$$

$\tilde{G}=\left\{\left(\mathrm{e}_{1},\left\{\mathrm{P}_{1}, \mathrm{P}_{6}, \mathrm{P}_{7}, \mathrm{P}_{10}\right\}\right),\left(\mathrm{e}_{2},\left\{\mathrm{P}_{2}, \mathrm{P}_{5}, \mathrm{P}_{8}\right\}\right),\left(\mathrm{e}_{3},\{\right.\right.$ $\left.\left.\mathrm{P}_{4}, \mathrm{P}_{9}, \mathrm{P}_{10}\right\}\right),\left(\mathrm{e}_{4},\left\{\mathrm{P}_{1}, \mathrm{P}_{2}, \mathrm{P}_{3}\right\}\right)$,

Then

$$
\left.\left(\mathrm{e}_{5},\left\{\mathrm{P}_{5}, \mathrm{P}_{6}, \mathrm{P}_{7}\right\}\right),\left(\mathrm{e}_{6},\left\{\mathrm{P}_{1}, \mathrm{P}_{4}, \mathrm{P}_{7}, \mathrm{P}_{9}\right\}\right)\right\} \text {. }
$$

$\tilde{\tau}=\{\widetilde{\emptyset}, \tilde{F}, \tilde{G}, \tilde{F} \sqcap \tilde{G}, \tilde{F} \sqcup \tilde{G}, \tilde{X}\}$ is a soft topology over $(\mathrm{U}$, $\mathrm{E}\}$ where $\mathrm{E}=\left\{\mathrm{e}_{1}, \mathrm{e}_{2}, \mathrm{e}_{3}, \mathrm{e}_{4}, \mathrm{e}_{5}, \mathrm{e}_{6}\right\}$.

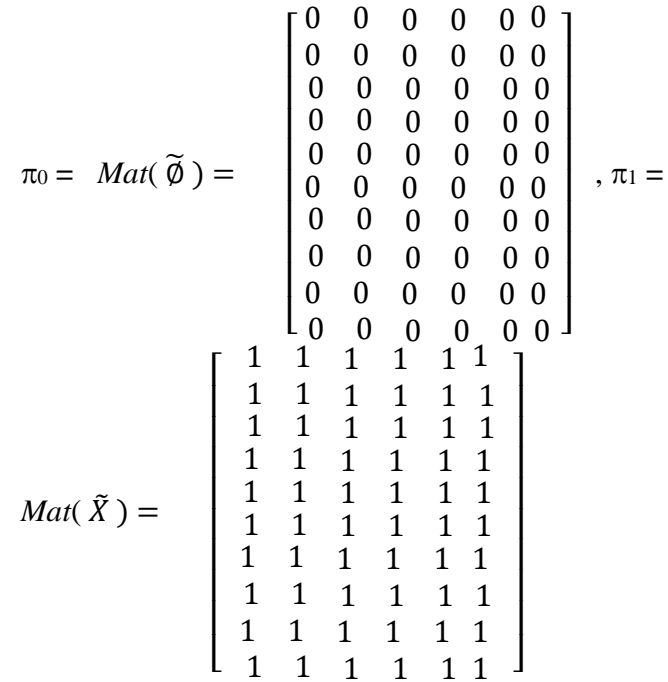

$\pi_{2}=\operatorname{Mat}(\tilde{F})=\left[\begin{array}{llllll}1 & 0 & 0 & 0 & 0 & 1 \\ 0 & 1 & 0 & 0 & 0 & 0 \\ 0 & 0 & 1 & 0 & 0 & 1 \\ 0 & 1 & 0 & 0 & 1 & 0 \\ 1 & 0 & 0 & 0 & 1 & 1 \\ 0 & 0 & 1 & 0 & 1 & 0 \\ 0 & 0 & 1 & 0 & 1 & 1 \\ 1 & 0 & 0 & 1 & 0 & 0 \\ 0 & 1 & 0 & 1 & 0 & 1 \\ 1 & 0 & 0 & 1 & 0 & 0\end{array}\right], \pi_{3}=$

$\left.\tilde{F} \sqcap \tilde{G}=\left\{\left(\mathrm{e}_{1},\left\{\mathrm{P}_{1}\right\}\right),\left(\mathrm{e}_{2},\left\{\mathrm{P}_{2}\right\}\right),\left(\mathrm{e}_{3}, \varnothing,\right\}\right),\left(\mathrm{e}_{4}, \varnothing\right\}\right)$, $\left.\left(\mathrm{e}_{5},\left\{\mathrm{P}_{5}, \mathrm{P}_{6}, \mathrm{P}_{7}\right\}\right),\left(\mathrm{e}_{6},\left\{\mathrm{P}_{1}, \mathrm{P}_{7}, \mathrm{P}_{9}\right\}\right)\right\}$

$\tilde{F} \sqcup \tilde{G}=\left\{\left(\mathrm{e}_{1},\left\{\mathrm{P}_{1}, \mathrm{P}_{5}, \mathrm{P}_{6}, \mathrm{P}_{7}, \mathrm{P}_{8}, \mathrm{P}_{10}\right\}\right),\left(\mathrm{e}_{2},\left\{\mathrm{P}_{2}, \mathrm{P}_{4}, \mathrm{P}_{5}, \mathrm{P}_{8}\right.\right.\right.$ , $\left.\left.\mathrm{P}_{9}\right\}\right),\left(\mathrm{e}_{3},\left\{\mathrm{P}_{3}, \mathrm{P}_{4}, \mathrm{P}_{6}, \mathrm{P}_{7}, \mathrm{P}_{9}, \mathrm{P}_{10}\right\}\right)$,

(e4, $\left.\left\{\mathrm{P}_{1}, \mathrm{P}_{2}, \mathrm{P}_{3}, \mathrm{P}_{8}, \mathrm{P}_{9}, \mathrm{P}_{10}\right\}\right),\left(\mathrm{e}_{5},\left\{\mathrm{P}_{4}, \mathrm{P}_{5}, \mathrm{P}_{6}\right.\right.$, $\left.\left.\left.\mathrm{P}_{7}\right\}\right),\left(\mathrm{e}_{6},\left\{\mathrm{P}_{1}, \mathrm{P}_{3}, \mathrm{P}_{4}, \mathrm{P}_{5}, \mathrm{P}_{7}, \mathrm{P}_{9}\right\}\right)\right\}$

Clearly 


$$
\begin{aligned}
& \pi_{4}=\operatorname{Mat}(\tilde{F} \sqcap \tilde{G})=\left[\begin{array}{cccccc}
1 & 0 & 0 & 0 & 0 & 1 \\
0 & 1 & 0 & 0 & 0 & 0 \\
0 & 0 & 0 & 0 & 0 & 0 \\
0 & 0 & 0 & 0 & 0 & 0 \\
0 & 0 & 0 & 0 & 1 & 0 \\
0 & 0 & 0 & 0 & 1 & 0 \\
0 & 0 & 0 & 0 & 1 & 1 \\
0 & 0 & 0 & 0 & 0 & 0 \\
0 & 0 & 0 & 0 & 0 & 1 \\
0 & 0 & 0 & 0 & 0 & 0
\end{array}\right], \pi_{5}= \\
& \operatorname{Mat}(\tilde{F} \sqcup \tilde{G})=\left[\begin{array}{ccccccc}
1 & 0 & 0 & 1 & 0 & 1 \\
0 & 1 & 0 & 1 & 0 & 0 \\
0 & 0 & 1 & 1 & 0 & 1 \\
0 & 1 & 1 & 0 & 1 & 1 \\
1 & 1 & 0 & 0 & 1 & 1 \\
1 & 0 & 1 & 0 & 1 & 0 \\
1 & 0 & 1 & 0 & 1 & 1 \\
1 & 1 & 0 & 1 & 0 & 0 \\
0 & 1 & 1 & 1 & 0 & 1 \\
1 & 0 & 1 & 1 & 0 & 0
\end{array}\right]
\end{aligned}
$$

Then $\mathrm{M}(\tilde{\tau})=\left\{\pi_{0}, \pi_{1}, \pi_{2}, \pi_{3}, \pi_{4}, \pi_{5}\right\}$. The following tables have been used to illustrate Theorem 3.5. That is Table 4.1 shows that $(\mathrm{M}(\tilde{\tau}), \oplus)$ is a Monoid with identity element $\pi_{0}$ and Table 4.2 shows that $(\mathrm{M}(\tilde{\tau}), \odot)$ is a Monoid with identity element $\pi_{1}$.

Table 4.1:

\begin{tabular}{|l|l|l|l|l|l|l|}
\hline$\oplus$ & $\pi_{0}$ & $\pi_{1}$ & $\pi_{2}$ & $\pi_{3}$ & $\pi_{4}$ & $\pi_{5}$ \\
\hline$\pi_{0}$ & $\pi_{0}$ & $\pi_{1}$ & $\pi_{2}$ & $\pi_{3}$ & $\pi_{4}$ & $\pi_{5}$ \\
\hline$\pi_{1}$ & $\pi_{1}$ & $\pi_{1}$ & $\pi_{1}$ & $\pi_{1}$ & $\pi_{1}$ & $\pi_{1}$ \\
\hline$\pi_{2}$ & $\pi_{2}$ & $\pi_{1}$ & $\pi_{2}$ & $\pi_{5}$ & $\pi_{2}$ & $\pi_{5}$ \\
\hline$\pi_{3}$ & $\pi_{3}$ & $\pi_{1}$ & $\pi_{5}$ & $\pi_{3}$ & $\pi_{3}$ & $\pi_{5}$ \\
\hline$\pi_{4}$ & $\pi_{4}$ & $\pi_{1}$ & $\pi_{2}$ & $\pi_{3}$ & $\pi_{4}$ & $\pi_{5}$ \\
\hline$\pi_{5}$ & $\pi_{5}$ & $\pi_{1}$ & $\pi_{5}$ & $\pi_{5}$ & $\pi_{5}$ & $\pi_{5}$ \\
\hline
\end{tabular}

Table 4.2:

\begin{tabular}{|l|l|l|l|l|l|l|}
\hline$\odot$ & $\pi_{0}$ & $\pi_{1}$ & $\pi_{2}$ & $\pi_{3}$ & $\pi_{4}$ & $\pi_{5}$ \\
\hline$\pi_{0}$ & $\pi_{0}$ & $\pi_{0}$ & $\pi_{0}$ & $\pi_{0}$ & $\pi_{0}$ & $\pi_{0}$ \\
\hline$\pi_{1}$ & $\pi_{0}$ & $\pi_{1}$ & $\pi_{2}$ & $\pi_{3}$ & $\pi_{4}$ & $\pi_{5}$ \\
\hline$\pi_{2}$ & $\pi_{0}$ & $\pi_{2}$ & $\pi_{2}$ & $\pi_{4}$ & $\pi_{4}$ & $\pi_{2}$ \\
\hline$\pi_{3}$ & $\pi_{0}$ & $\pi_{3}$ & $\pi_{4}$ & $\pi_{3}$ & $\pi_{4}$ & $\pi_{3}$ \\
\hline$\pi_{4}$ & $\pi_{0}$ & $\pi_{4}$ & $\pi_{4}$ & $\pi_{4}$ & $\pi_{4}$ & $\pi_{4}$ \\
\hline$\pi_{5}$ & $\pi_{0}$ & $\pi_{5}$ & $\pi_{2}$ & $\pi_{3}$ & $\pi_{4}$ & $\pi_{5}$ \\
\hline
\end{tabular}

The choice of the soft sets in the above discussion by a doctor depends upon the patients medical history. The doctor recommends that the patient $\mathrm{P}_{\mathrm{i}}$ has to choose compulsorily the parameter $\mathrm{e}_{\mathrm{j}}$ if and only if $\mathrm{P}_{\mathrm{i}} \in(\tilde{F} \sqcap \tilde{G})\left(\mathrm{e}_{\mathrm{j}}\right)$ that is if and only if the $(\mathrm{i}, \mathrm{j})^{\text {th }}$ entry in $\pi_{4}=\operatorname{Mat}(\tilde{F} \sqcap \tilde{G})$ is 1 . The doctor recommends that the patient $P_{i}$ has to choose optionally the parameter $\mathrm{e}_{\mathrm{j}}$ if and only if $\mathrm{P}_{\mathrm{i}} \in(\tilde{F} \sqcup \tilde{G})\left(\mathrm{e}_{\mathrm{j}}\right)$ that is if and only if the $(\mathrm{i}, \mathrm{j})^{\text {th }}$ entry in $\pi_{4}=\operatorname{Mat}(\tilde{F} \sqcup \tilde{G})$ is 1. If the row corresponding to the patient $P_{i}$ has more than one 1's in $\operatorname{Mat}(\tilde{F} \sqcap \tilde{G})$ or in $\operatorname{Mat}(\tilde{F} \sqcup \tilde{G})$ then the doctor advised the right parameter according to the medical history of the patient. The following algorithm may be used by a doctor for his recommendations.

\section{Algorithm 4.3:}

1. Input $U$, the universe set of patients.

2. Input E, a set of parameters for recommendations.

3. Input the soft sets $\tilde{F}$ and $\tilde{G}$.
4. Input $\operatorname{Mat}(\tilde{F})$ and $\operatorname{Mat}(\tilde{G})$.

5. Compute $\mathrm{A}=\operatorname{Mat}(\tilde{F}) \odot \operatorname{Mat}(\tilde{G})=\left[\mathrm{a}_{\mathrm{ij}}\right]$.

6. Compute $\mathrm{B}=\operatorname{Mat}(\tilde{F}) \oplus \operatorname{Mat}(\tilde{G})=\left[\mathrm{b}_{\mathrm{ij}}\right]$.

7. Input the patient $\mathrm{P}_{\mathrm{i}}$.

8. If $a_{i j}=1$ for some $j$, recommend $e_{j}$ to $P_{i}$.

9. If $a_{i j}=0$ for all $\mathrm{j}$, go to 10 .

10. If $b_{i j}=1$ for some $j$, recommend $e_{j}$ to $P_{i}$.

\section{Conclusion}

A soft set is represented by a 0-1 matrix that is used to characterize soft topology. A binary data set consisting of binary matrices has been created by using a soft topology on the list of diabetic patients and this data set may be used by a doctor for the recommendations to the patients personally or virtually. In this regard, appropriate algorithm is also given for implementing the discussion.

\section{Acknowledgement}

The first author thank the management of Erode Arts and Science College, India and $R \& D$ wing of Bharathiar University, India for giving permission to do research in this area of study.

\section{References:}

[1] Abdul Iguda, Soft Sets and Soft topological Notions, Journal of new theory, 30(2020), 21-34.

[2] Baravan A. Asaad, Tareq M. Al-shami, Abdelwaheb Mhembi, Bioperators on soft topological spaces, AIMS Mathematics , 6(11)(2021), 12471-12490.

[3] Bashir Ahmad and Sabir Hussain, On some structures of soft topology, Mathematical Sciences, (2012), $1-7$.

[4] Evanzalin Ebenanjar .P and Thangavelu.P, Between Nearly open sets and Soft $1-5$.

Nearly open sets, Appl. Math. Inf. Sci. 10(6)(2016),

[5] Maji P K , Roy A R and Biswas R, An Application of Soft Sets in a Decision Making Problem, Computrers and Mathematics with Applications 44(2002), 1077-1083

[6] Molodtsov.D , Soft Set Theory - First Results, Computers and Mathematics with Applications, 37(1999), 19-31.

[7] Muhammed Saeed, Manzoor Hussain, Abdul Aleem Mughal, A study of soft sets with soft members and soft elements:A new approach, Punjab university of mathematics 52(8)(2020), 1-15.

[8] Muhammad Shabir \& Munazza Naz 2011,' On Soft Topological Spaces', Comput. Math. Appl., 61(2011), 1786-1799.

[9] Peyghan.E, Samadi.B , Tayebi.A , About soft topological spaces, Journal of New Results in Science, 2(2013), 60-75. 
[10] Pawlak Z, Rough sets, International Journal of Information and Computer Sciences, 11(1982), 341-356.

[11] Saeid Jafari, Abd El-Atik, Raja M. Latif, M.K. El.Bably, Soft topological spaces induced via soft relations, WSEAS Transactions on mathematics 2020, doi:10.37394/23206.2021.20.1

[12] Samer Al Ghour, Soft $w_{p}$-open sets and soft $w_{p}-$ continuity in soft topological spaces, Mathematics 9(2021), 2632.

https://doi.org/10.3390/math9202632

[13] Shabir Hussain and Bashir Ahmad, Some properties of soft topological spaces, Computers and Mathematics with Applications, 62(2011), 40584067.

[14] Tareq M.Al-shami, Bipolar soft sets:Relations between them and ordinary points and their applications, complexity vol 2021, 2021, 14 pages.

[15] Tugce Aydin, Serdar Enginoglu, Some results on soft topological notions, Journal on new results in science 10(1)(2021), 65-75. 